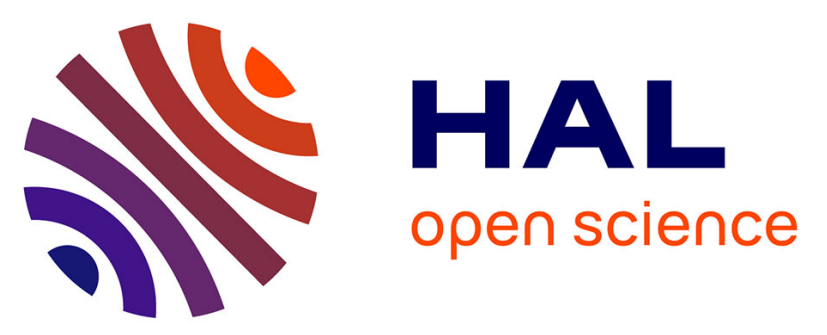

\title{
LA LOI D'ACTION DE LA PRÉSURE DANS LA COAGULATION DU COMPLEXE CASÉINATE DE CALCIUM + PHOSPHATES DE CALCIUM
}

C. Christen, E. Virasoro

\section{- To cite this version:}

C. Christen, E. Virasoro. LA LOI D'ACTION DE LA PRÉSURE DANS LA COAGULATION DU COMPLEXE CASÉINATE DE CALCIUM + PHOSPHATES DE CALCIUM. Le Lait, 1932, 12 (119), pp.923-931. hal-00895081

\section{HAL Id: hal-00895081 https://hal.science/hal-00895081}

Submitted on 1 Jan 1932

HAL is a multi-disciplinary open access archive for the deposit and dissemination of scientific research documents, whether they are published or not. The documents may come from teaching and research institutions in France or abroad, or from public or private research centers.
L'archive ouverte pluridisciplinaire HAL, est destinée au dépôt et à la diffusion de documents scientifiques de niveau recherche, publiés ou non, émanant des établissements d'enseignement et de recherche français ou étrangers, des laboratoires publics ou privés. 


\section{LA LOI D'ACTION DE LA PRÉSURE DANS LA COAGULATION DU COMPLEXE CASÉINATE DE CALCIUM + PHOSPHATES DE CALCIUM (1)}

par

\section{CHRISTEN}

Ingénieur chimiste

Institut de Recherches scientifiques et techniques. et

\author{
E. VIRASORO \\ Ingénieur chimiste \\ Chef de travaux au Laboratoire \\ de Chimie-Physique.
}

Travail de l'Institut de Recherches scientifiques et techniques de la Faculté de Chimie industrielle, Université Nationale du Littoral, Santa-Fé, République Argentine.

Quand on ajoute l'enzyme présure ou lab au lait, celui-ci subit certaines modifications qui se terminent par une coagulation. Si l'on fait varier la proportion de présure ajoutée, le temps mis pour arriver à la coagulation varie également.

SEGELCKE et STORCH, étudiant cette réaction enzymatique, ont émis la loi suivante : "A une même température, le produit de la concentration en ferment par le temps de coagulation est constant. "

Des travaux postérieurs d'autres auteurs, parmi lesquels : Soxhlet, Lörcher, Lenk, etc., ont confirmé cette loi dans une première approximation, en trouvant qu'elle se vérifie, mais dans des limites étroites, variables d'ailleurs pour chacun d'eux. En général, ils ont trouvé que les produits diminuent quand la concentration en présure diminue.

Parmi les auteurs plus récents, Grimmer et KRÜGER ont trouvé aussi que cette loi se vérifie, mais entre des limites toujours très étroites, en faisant remarquer que les produits représentent une fonetion exponentielle de la quantité d'enzyme et qu'ils suivent, en conséquence, une courbe Iogarithmique.

Ch. Porcher [1] pense que ces anomalies pourraient être dues aux erreurs qu'on commet dans la fixation du moment exact de la coagulation et par suite dans l'appréciation du temps. En effet, certains expérimentateurs fixent comme point de coagulation, des moments de la réaction très différents les uns des autres, et se contentent de mesurer les temps en minutes ou en demi-minutes.

Nous nous sommes proposé, dans ce travail, de contribuer à l'éclaircissement de la loi d'action de la présure. Pour cela, nous avons cru convenable d'opérer sur un liquide de constitution plus simple que le lait même.

Le lait naturel n'est pas un liquide de composition constante et il est sujet, en outre, à des variations dues à divers facteurs, parmi

(1) Paru dans le Volume jubilaire du Professeur Ch. Porcher. 
lesqueIs le plus commun est celui des ferments. La réaction du milieu, la concentration en sels alcalins et alcalino-terreux, la concentration en ions calcium, la proportion d'albumine et de globuline, la quantité de caséine présente, la teneur en anhydride carbonique, etc., sont des facteurs qui influencent également l'action de la présure sur le lait.

De ces différents facteurs, les uns agissent d'une façon favorable sur le processus de la coagulation, tandis que les autres le retardent en jouant un rôle protecteur vis-à-vis de la caséine.

Puisque le lait est un liquide d'une composition si complexe, il n'est pas nécessaire de faire ressortir le grand avantage que l'on retire d'étudier la loi de l'emprésurage sur la partie du lait qui est touchée directement dans ce phénomène : le complexe cxséinate + phosphates de chaux. Pour ce, nous nous sommes inspirés des notables travaux de $\mathrm{Ch}$. Porcher relevant du recours à la méthode synthétique dans l'étude du lait.

Nous nous sommes proposé d'éviter toute cause d'erreur qui pourrait influencer les résultats.

En ce qui concerne la présure, nous avons employé pour chaque série de déterminations, une même concentration, telle que, ajoutée au complexe, la solution de celui-ci augmentait au maximum de $6 \%$. Les mesures des temps ont été effectuées à la seconde et la température réglée au 1/10 de degré centigrade.

Ce que nous estimons être dominant dans ce travail, e'est la détermination du point de coagulation. Il est souvent difficile de l'apprécier, parce qu'il n'est pas possible de déterminer, à l'œil nu, le moment exact où la caséine, complètement dédoublée, commence à gélifier sous l'influence des ions calcium.

La méthode la plus généralement suivie consiste à observer la formation des flocons. Pour des emprésurages rapides, l'erreur absolue dans la mesure du temps est sans doute petite, mais relativement au temps que demande la coagulation, elle devient appréciable A mesure que les emprésurages se font avec des concentrations plus faibles de présure, la détermination du moment exact de la coagulation se fait chaque fois plus difficile. Les erreurs sont encore plus grandes quand on prend comme point de coagulation le moment où la gélification est arrivée à un tel état que, par renversement du tube à essais dans lequel on a réalisé l'expérience, le coagulum ne tombe pas.

LE PRINOIPE DE NOTRE TECHNIQUE. - Nous avons alors cherché une propriété qui subisse, au moment vrai de la coagulation, des variations telles qu'elles puissent nous servir pour fixer avec plus de précision ce moment même. Nous avons obtenu de bons résultats avec la viscosité.

La viscosité ne nous fournit pas un chiffre duquel on puisse tirer une conclusion touchant directement le point de coagulation que nous 
cherchons, mais sa variation est un excellent indicateur des modifications que subit le liquide et cela nous a été d'une grande utilité.

Une observation sans aucune interruption de la viscosité pendant toute la durée de l'emprésurage nous a montré que, depuis le moment où l'on a mis en présence la présure et le liquide sur lequel elle va agir, cette propriété demeure pratiquement constante jusqu'à un certain moment, où elle augmente de valeur, en s'accentuant fortement vers la fin.

$\mathrm{Si}$, sur des axes de coordonnées, nous mettons les valeurs des viscosités en ordonnées et les temps en abscisses, on obtient des courbes de l'allure indiquée dans les graphiques I et II.

En recourant à des essais comparatifs basés sur l'observation à simple vue du point de coagulation dans des emprésurages à temps réduits, pour lesquels la fixation de ce point se fait avec facilité, nous avons constaté que cette augmentation brusque de viscosité correspond à l'apparition des flocons. Dans les emprésurages de longue durée, dans lesquels l'observation à simple vue rend difficile la précision du point de coagulation, Ia variation brusque de la viscosité nous a servi, de même que pour ceux de durée réduite, à fixer ce point.

Il n'y a pas de doute que les points de coagulation fournis par cette méthode sont plus précis ; ils sont comparables entre eux et sont moins sujets aux erreurs personnelles d'observation.

L'APPAREIL. - Les déterminations de la viscosité exigent un viscomètre continu qui puisse indiquer sans interruption l'allure des variations de la viscosité depuis le début de l'emprésurage jusqu'à la fin.

Dans ce but, nous avons adopté le viscomètre rotatif électromagnétique imaginé par notre collègue de cet Institut, l'ingénieur C. G. Berraz [2]. En principe, il s'agit d'un cylindre lesté, en verre, B (voir figure), immergé dans le liquide à coaguler, contenului-même dans un récipient, $\nabla$, fixé à un axe creux vertical, $E$, lequel tourne à une vitesse constante (12 révolutions-minute dans nos expériences) grâce à un moteur. Pendant que le récipient V tourne, le frottement du liquide engendre un couple de torsion proportionnel à la viscosité sur le cylindre B et dont l'action se transmet par la baguette de suspension $\mathrm{E}$ à un système astatique, I, constitué par des petits aimants permanents. Deux spires en cuivre, C, en série, en position normale aux aimants, créent un champ qui tend à faire tourner le système astatique, en éloignant les aimants dans un sens contraire à eelui déterminé par le liquide. Des arrêts, convenablement disposés, empêchent que le système suspendu bifilairement puisse tourner.

On part d'une position d'équilibre déterminée optiquement au moyen d'un miroir, M, et d'un signe de référence. Pour effectuer une mesure, on compense le couple de frottement par un couple électro- 
magnétique, c'est-à-dire qu'on porte le système à la position initiale d'équilibre en réglant le courant d'une pile au moyen d'un rhéostat approprié.

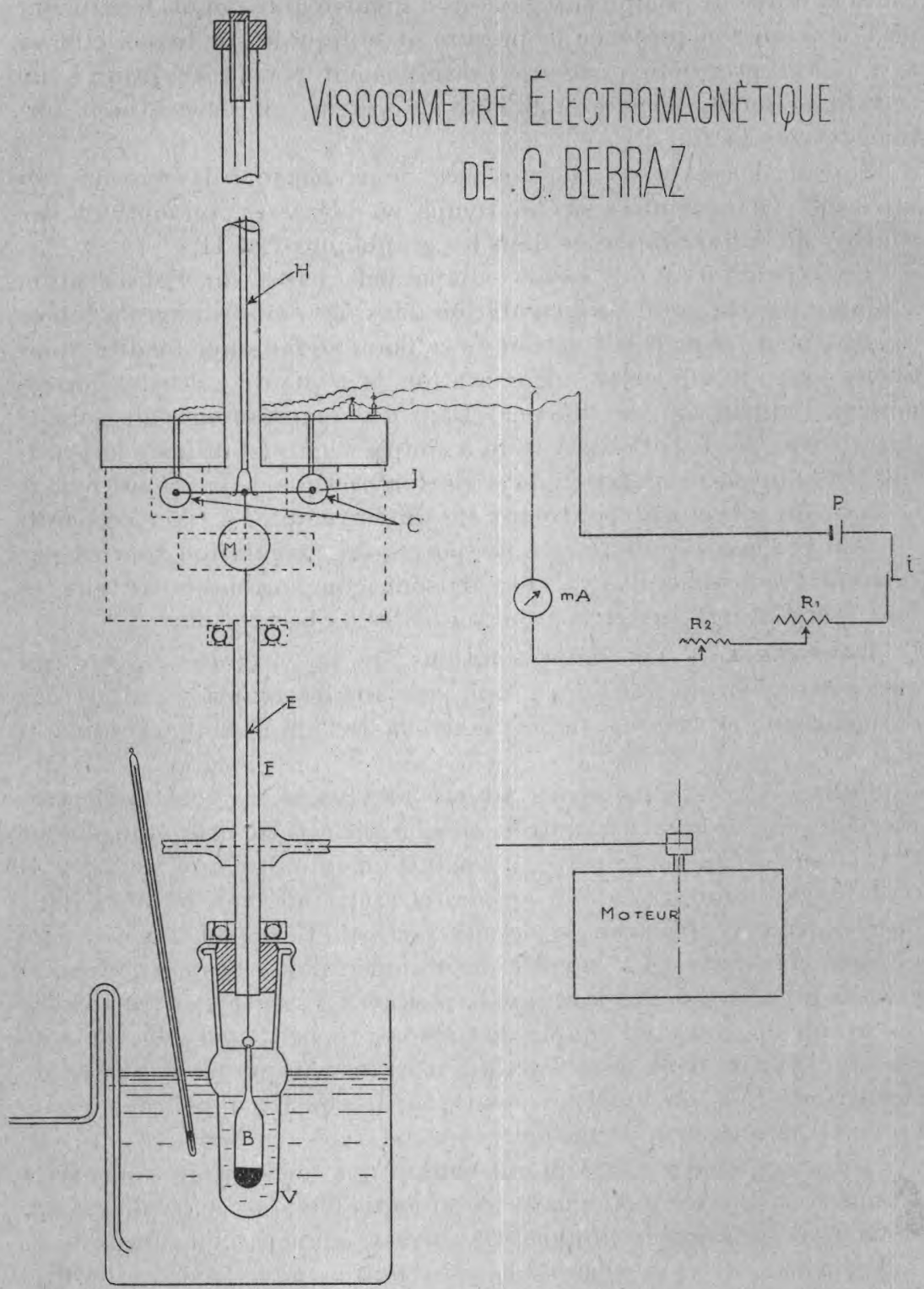

Une fois obtenue la position d'équilibre, on lit l'intensité du courant dans un milliampèremètre intercalé dans le circuit. D'après les 


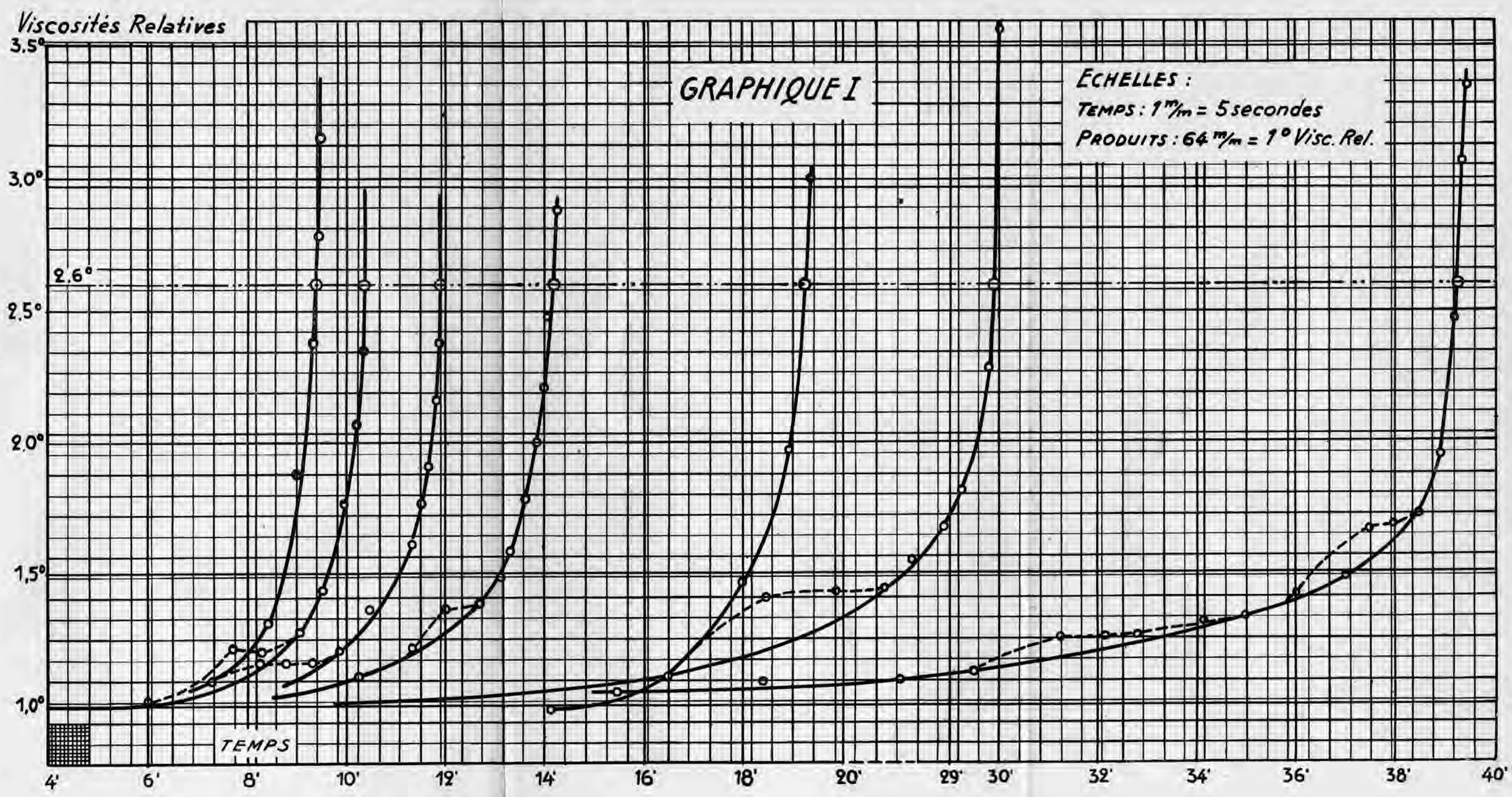




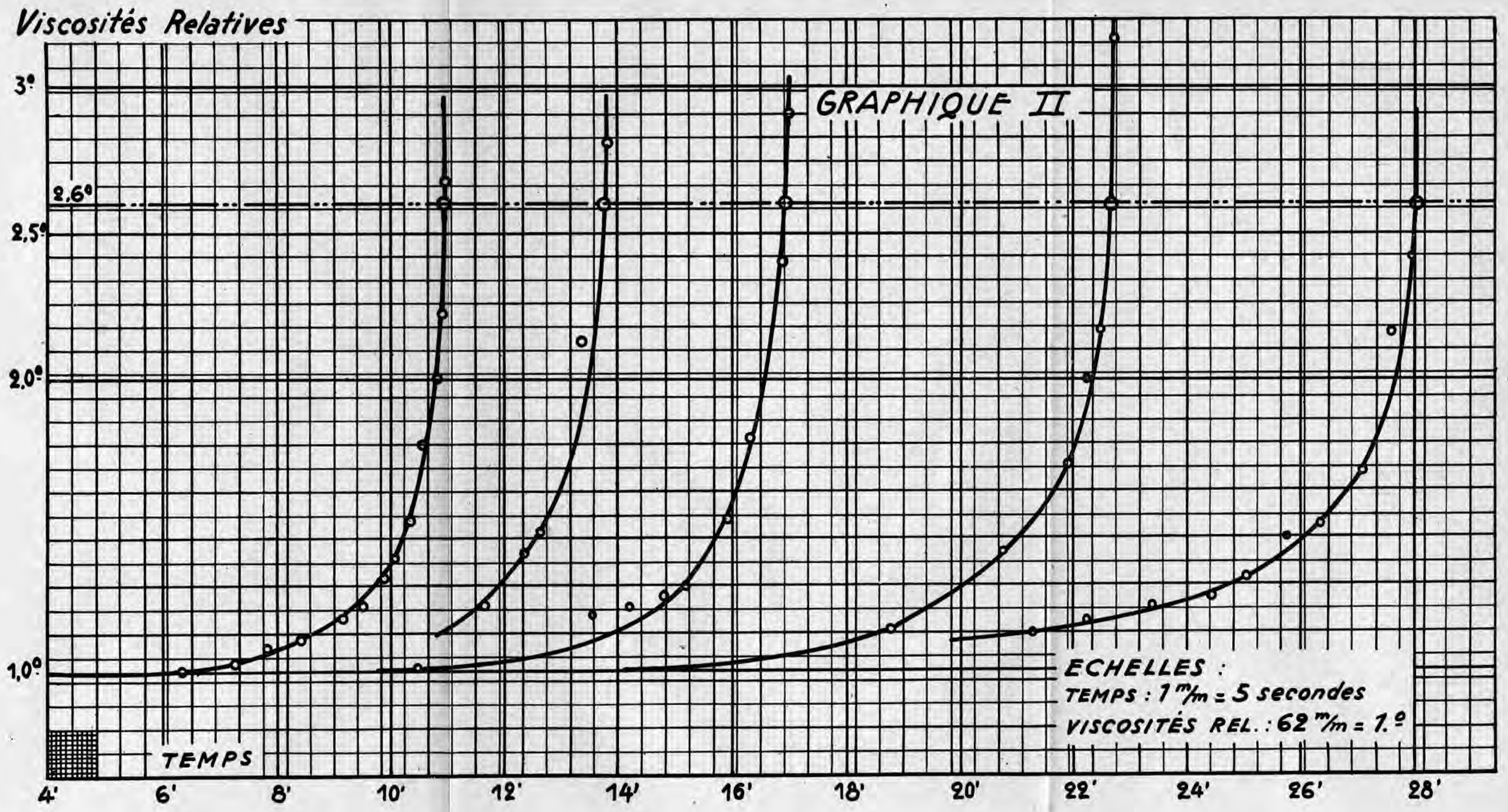


Viscosites Relatives

3

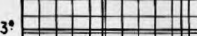

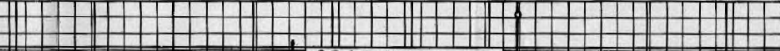

GRAPHIQUE II

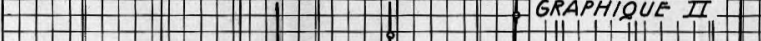

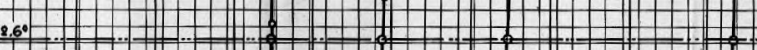

2,5 .

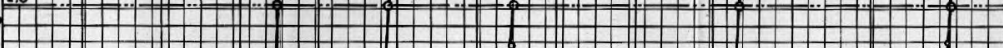

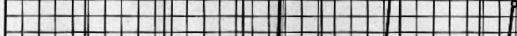

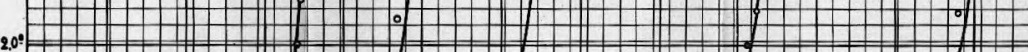

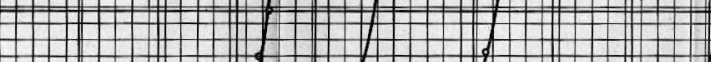

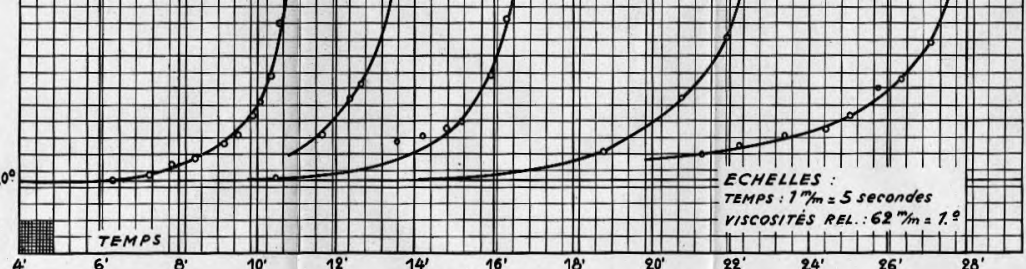


essais de l'auteur, l'intensité est proportionnelle à la viscosité du liquide.

Le récipient $V$ est immergé dans un thermostat; réglé au 1/10 de degré centigrade.

Pour chaque détermination de viscosité, on fait la lecture de l'intensité du courant dans le milliampèremètre, de temps en temps au commencement, puis, aussitôt que l'intensité s'élève, les lectures sont faites à intervalles plus rapprochés et cela jusqu'au moment où il devient impossible de le faire à cause des trop rapides variations, ce qui d'ailleurs n'a aucun intérêt pour ces sortes d'expériences.

Si on porte en abscisses les temps en secondes et en ordonnées les viscosités relatives correspondantes, on obtient, pour chaque série d'essais, une famille de courbes de l'allure indiquée dans les graphiques I et II. Nous avons fixé, comme point de coagulation, le temps correspondant à une viscosité relative de 2,6 , point moyen d'une petite zone, laquelle, pour des temps relativement réduits, coïncide avec le point observé à simple vue. De cette façon, l'erreur maximum commise est de 5 secondes pour des emprésurages longs; I'erreur relative pour les emprésurages de 40 minutes par exemple n'est que de $2 \%$.

PREPARATION de LA CAsÉine pURE. - La caséine pure, nécessaire à la. préparation du complexe, a été obtenue en nous basant sur la technique de Ch. Porcher [3].

Dans une cuve en grès de 100 litres, on met 10 litres de lait passé trois fois à la centrifuge et on le dilue avec 40 litres d'eau distillée; on ajoute $\mathrm{HCl} \mathrm{N} / 2$ très lentement, en mẹme temps que le liquide est agité. On interrompt l'addition d'acide quand le $p H$, qui est contrôlé au moyen d'un potentiomètre, arrive au point isoélectrique de la caséine $(p H=4,6)$. Le sérum est séparé par décantation et la caséine passée à une essoreuse, où elle est lavée avec de l'eau distillée. La masse de caséine est mise en suspension dans de l'eau distillée au moyen d'un agitateur et passée de nouveau à l'essoreuse, où elle est lavée à l'eau distillée. Cette opération est répétée encore trois fois.

La caséine ainsi séparée est dissoute dans $\mathrm{NH}^{*}$, en la portant préalablement en suspension dans environ 10 litres d'eau distillée et en ajoutant l' $\mathrm{NH}^{\circ} \mathrm{d}^{\mathrm{u}}$ une concentration $\mathrm{N} / 2$, très lentement et par agitation énergique de la suspension, jusqu'à ce que le $p H$ du caséinate soit de 6,9 à 7,0 .

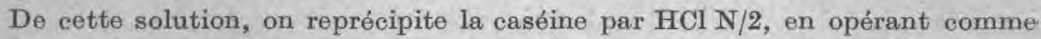
l'on a fait pour la séparer du lait écrémé.

Les redissolutions et reprécipitations sont répétées encore trois fois, de façon que finalement on ait réalisé quatre précipitations et trois dissolutions à partir du lait.

La masse de caséine obtenue dans la dernière précipitation est lavée à fond dans l'essoreuse jusqu'à ee que l'eau de lavage donne une réaction négative avec le réactif de Nessleer.

Pour déshydrater cette caséine, on la broie dans un mortier avee l'alcool, en 
la laissant jusqu'au lendemain. L'aleool est séparé par filtration au vide. On répète ce traitement alcoolique encore trois fois.

La galette de caséine de la dernière filtration sur le vide est broyée avec l'éther et traitée comme on l'a fait avec l'alcool. La caséine est étendue sur des' plats mis dans un exsiccateur où on fait le vide; ces plats sont passés finalement dans une étuve à la température de $35-40^{\circ} \mathrm{C}$., avec eireulation d'air, jusqu'à élimination de l'odeur d'éther.

Ce produit préparé comme on vient de l'indiquer a une teneur en cendres de $0,51 \%$ et une humidité de $8,30 \%$. D'après le dosage de l'azote, la pureté en protéine est de $90,99 \%$.

preparation du complexe. - Ch. Porcher [4] fixe le contenu en easéine dans le lait, pour la préparation du complexe, à $3 \%$; la quantité de $\mathrm{CaO}$ qui lui correspond est de 0 gr. 110, en partie combinée à la protéine sous la forme de caséinate de chaux, et le restant combiné à l'acide phosphorique en donnant les phosphates de chaux.

En nous basant sur ces données, nous avons préparé un caséinate de chaux très alcalin, caséinate "à 1100 ", comme le dit le savant cité ; l'excès de $\mathrm{CaO}$ est neutralisé par $\mathrm{PO}^{4} \mathrm{H}^{3}$, jusqu'à atteindre un $p \mathrm{H}$ très voisin du $p \mathrm{H}$ normal du lait.

On a opéré ainsi : D'après la teneur en protéine de la caséine, eompte tenu done de l'humidité, pour $100 \mathrm{~cm}^{3}$ de complexe, on pèse $3 \mathrm{gr} .30$, qu'on broie dans un mortier avec quelques $\mathrm{cm}^{3}$ d'eau distillée bouillie, en formant une masse qui est versée dans une cuve en verre, où l'on ajoute un lait de chaux en suspension très fine, presque translucide, d'une concentration variant entre $1 \mathrm{gr} .17 \mathrm{et}$ $1 \mathrm{gr} .22 \%$, en même temps qu'on agite énergiquement au moyen d'un agitateur électrique à baguette nickelée. (Nous avons utilisé avec un excellent résultat un agitateur construit spécialement pour préparer le cocktail et qu'on peut acquérir dans le commerce. Cet agitateur donne 6.000 tours à la minute, pouvant être réduit à volonté en intercalant en série un rhéostat dans le circuit.)

On laisse ce caséinate "mûrir " k̀ basse température jusqu'au lendemain. A ce moment, le $p \mathrm{H}$ a une valeur comprise entre 9,4 et 9,5 . Ensuite on ajoute $\mathrm{PO}^{4} \mathrm{H}^{3}$ à une concentration d'environ $2 \%$, ce qui se fait goutte à goutte pendant qu'on agite le caséinate énergiquement avec l'agitateur déjà cité ; cette opération est interrompue quand le $p \mathrm{H}$ a une valeur de 6,5 environ.

Le complexe ainsi préparé est conservé à basse température juisqu'au moment de commencer une série d'essais.

Nous avons constaté que pour obtenir un complexe caillant normalement avec la présure, il est nécessaire de réaliser la neutralisation par l'acide phosphorique à une température comprise entre $10^{\circ}$ et $20^{\circ} \mathrm{C}$.

Si le $p H$ du complexe a une valeur trop élevée : 6,8, par exemple, le coagulum obtenu par emprésurage n'est pas homogène et ne se prend pas bien en masse, sauf pour les concentrations élevées en présure, qui 
le font cailler en moins de 5 minutes. C'est à cause de cela qu'on a adopté un $p \mathrm{H}$ compris entre 6,4 et 6,5 .

Nous avons observé aussi que la " maturation " du caséinate est nécessaire pour obtenir un bon complexe.

LA PRÉsure. - La présure employée dans ces expériences est de la présure en poudre d'origine allemande (marque Blumenthal), d'une force telle que $1 \mathrm{gr}$. est eapable de coaguler 100 litres de lait dans les conditions courantes.

En partant de cette présure, nous avons préparé des solutions en en pesant un certain poids et en la portant à un volume déterminé avec de l'eau distillée ; la solution est ensuite filtrée sur papier à filtre. Elle présente une réaction neutre.

Nous avons constaté que Ia température et la lumière altèrent rapidement l'activité de cette enzyme. A la température ambiante $\left(30^{\circ}\right.$ à $35^{\circ} \mathrm{C}$. en été) et sans la préserver de la lumière solaire, l'activité est presqué complètement détruite en 3-4 heures. Pour cette raison, aussitôt que la solution de présure est préparée, on la verse dans un gros tube à essais, lequel est conservé dans un thermostat contenant de la glace et bien couvert. En opérant de cette façon, on a observé que l'activité de la solution de présure n'a subi aucune variation, tout au moins dans le temps nécessaire pour une série d'essais, ce qu'on vérifiait en répétant, à la fin d'une des susdites séries et dans les mêmes conditions, la première détermination; la variation trouvée a toujours été comprise dans les limites des erreurs d'expériences admissibles.

LE MODE OPÉrATOIRE. - Pour chaque détermination, on verse dans un tube à essais $15 \mathrm{~cm}^{3}$ du complexe, mesuré à la burette, toujours à la même température, et on le place dans le thermostat du viscosimètre à la température de $35^{\circ} \mathrm{C}$.

A part, on pèse, au moment de réaliser l'emprésurage, la quantité de solution de présure nécessaire et on la place dans le même bain. Aussitôt que les deux récipients ont atteint la température du bain, on le mélange rapidement en les versant l'un dans l'autre, deux à trois fois, et on commence à compter le temps à partir du moment où l'on a versé la première fois le complexe sur la solution de présure. On remplit de suite le récipient $B$ du viscosimètre, lequel était immergé jusqu'à ce moment dans le thermostat, et on le fixe à l'appareil.

Pour la sûreté dans les résultats, chaque détermination a été répétée une deuxième fois ; on a toujours eu des concordances acceptables.

Ci-dessous nous présentons le tableau des valeurs correspondant à deux séries d'essais, choisies parmi celles qu'on a réalisées avec le complexe, et une série effectuée avec du lait écrémé, pour servir de comparaison. 


\section{Courbe $n^{\circ} 1$ :}

Essai avec du lait écrémé.

Acidité : $21^{\circ} \mathrm{D}$.

Température de l'expérience : $35^{\circ} \mathrm{C}$.

Concentration de la solution de présure : $0,05 \%$.

Temps en secondes

427
643
815
941

1.833

3.405

\begin{tabular}{|c|c|}
\hline \multicolumn{2}{|c|}{$\begin{array}{l}\text { Quantité de présure } \\
\text { dans } 100 \mathrm{~cm}^{3} \text { de lait }\end{array}$} \\
\hline 12,5 & gr: sol. $0,5 \%$ \\
\hline 8,75 & D \\
\hline 6,25 & 》 \\
\hline 5,00 & 》 \\
\hline 2,50 & D. \\
\hline 1,25 & » \\
\hline
\end{tabular}

Produit

5.337

5.626

5.093

4.705

4.582

4,256

\section{Courbe no 2 :}

Essai avec le complexe.

$p \mathrm{H}$ du complexe : 6,4.

Température de l'expérience : $35^{\circ} \mathrm{C}$.

Concentration de la solution de présure : 0,05\%.

Temps en secondes

$$
360
$$

565

625

715

855

1.157

1.795

2.357
Quantité de présure
dans $100 \mathrm{~cm}^{3}$ de lait

1,50 gr. sol.

1,00 ,

0,90 》

0,80 "

0,70 》

0,55 "

0,37 "

0,30 "
Produit

540

565

562,5

572

598,5

636,3

664,1

707,1

\section{Courbe $n^{\circ} 3$ :}

$p \mathrm{H} d u$ complexe : 6,32 .

Température de l'expérience : $35^{\circ} \mathrm{C}$.

Concentration de la solution de présure : $0,025 \%$.

Temps en secondes

660

828

1.020

1.360

1.685
Quantité de présure dans $100 \mathrm{~cm}^{3}$ de lait

$$
0,40 \text { gr. sol. }
$$

0,35

0,30

0,23

0,20
Produit

264

289,8

306

312,8

336

\section{GONGLUSIONS.}

Les valeurs obtenues avec le lait écrémé, lesquelles d'ailleurs sont en concordance avec celles de la plupart des auteurs, sont opposées avec celles qu'on a trouvées avec le complexe. Pour celui-ci, les produits de la concentration par le temps augmentent graduellement et 


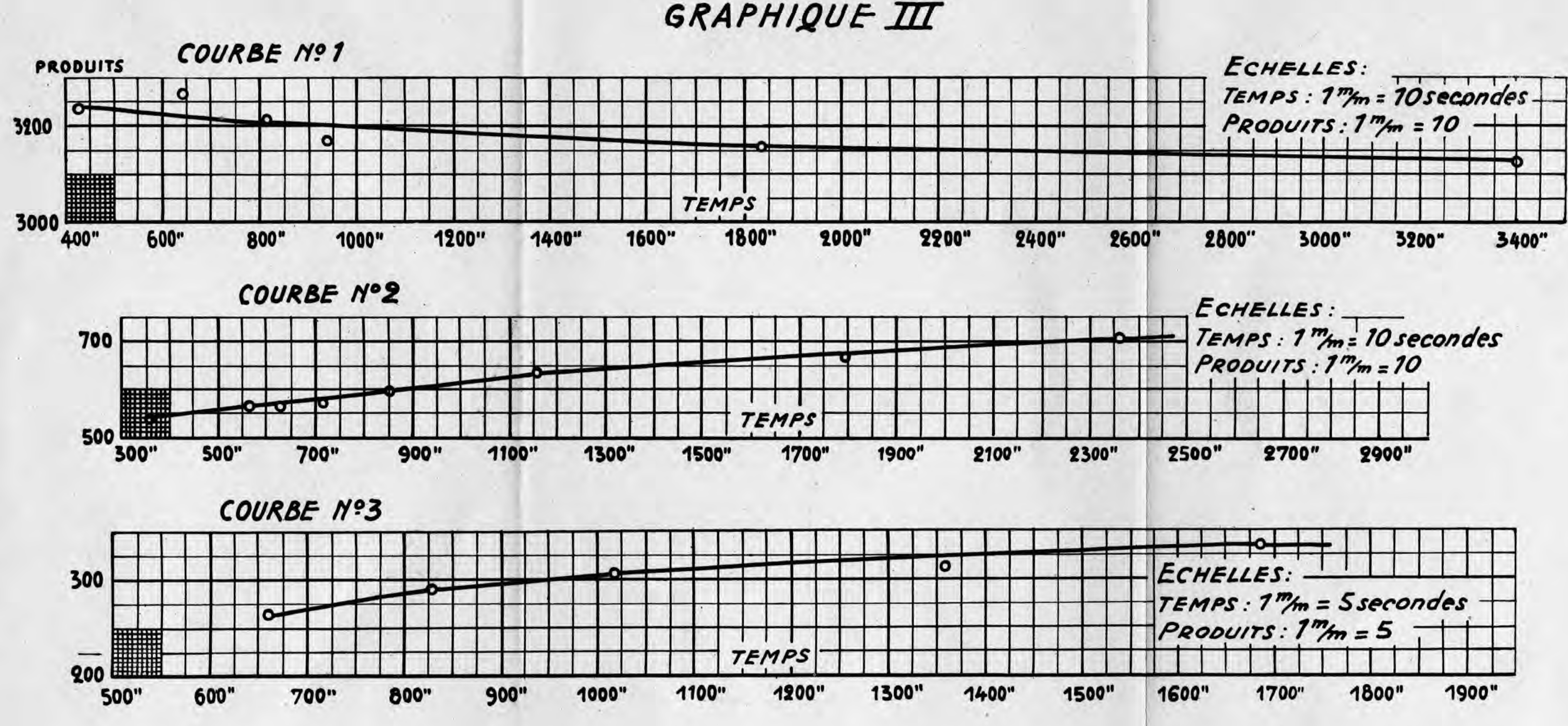




\section{GRAPHIQUE III}

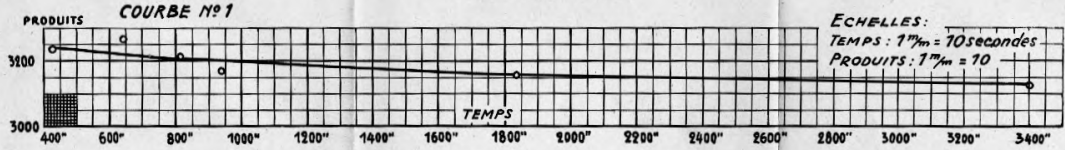

COURBE $N^{\circ} 2$

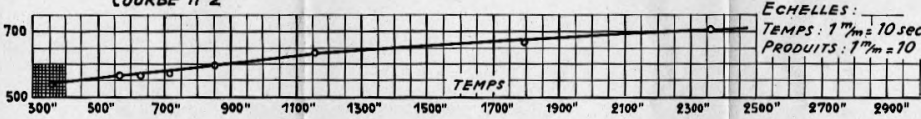

COURBE N:3

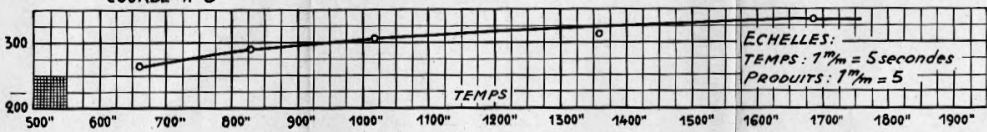


lentement au fur et à mesure que la concentration de la présure diminue.

Le parallélisme qu'on observe entre les deux courbes des expériences avec le complexe, et qui d'ailleurs correspondent à des écarts différents de temps, nous montrent la remarquable régularité du phénomène étudié.

En conséquence, on peut conclure, dans une première approximation, que la loi de SEGELCKE et STORCH ne se vérifie pas avec le complexe caséinate de chaux + phosphates de chaux, dans les limites des temps expérimentés. Nous croyons intéressant de poursuivre ce travail en essayant de déterminer le rôle de chaque constituant du lait dans la coagulation par la présure.

\section{BIBLIOGRAPHIE.}

[1] Ch. Porcher, Le lait au point de vue colloïdal, p. 246.

[2] G. Berraz. Viscosimètre rotatif électromagnétique. Annales de l'Institut de Recherches scientifiques et techniques, Faculté de Chimie industrielle de Santa-Fé (sous presse).

[3] Ch. Porcher. Le lait au point de vue colloïdal, p. 160.

[4] Loc. cit., p. 350.

\section{RECHERCHES SUR LA CONSTANTE MOLÉCULAIRE SIMPLIFIEEE (C. M. S.) DE CERTAINS LAITS : \\ LAITS D'AVORTEMENT, DE VACHES « EN CHALEUR \# ET DE VACHES HOLLANDAISES ( 1 ) \\ par}

le Dr A. LATAIX

Vétérinaire-Capitaine.

"Contradiction est une mauvaise marque de vérité. Plusieurs choses certaines sont contredites, plusieurs fausses passent sans contradiction : ni la contradiction n'est marque de fausseté, ni l'incontradiction n'est marque de vérité. "

Pascat.

Voici l'exposé des faits qui nous ont déterminé à faire quelques recherches sur la C. M. S. de certains laits.

Un prélèvement de lait a été fait le 5 juin 1929 chez M. H... Il y a eu une analyse administrative et une expertise contradictoire, qui ont donné les résultats suivants :

(1) Paru dans le Volume jubilaire du Professeur Ch. Porcher. 